\title{
The impact of preoperative frailty status on outcomes after transcatheter aortic valve replacement
}

\section{An update of systematic review and meta-analysis}

\author{
LiLi Huang, MDª , XiaoShuang Zhou, MDª XiaoYun Yang, MD ${ }^{b, *}$, Hai Yu, MD ${ }^{a, *}$
}

\begin{abstract}
Background: Frailty is a syndrome of impaired physiologic reserve and decreased resistance to stressors and can often be seen in high-risk patients undergoing transcatheter aortic valve replacement (TAVR). Preoperative frailty status is thought to be related to adverse outcomes after TAVR. We conducted this systematic review and meta-analysis to determine the impact of preoperative frailty status on outcomes among patients after TAVR.

Methods: PubMed, Embase, and the Cochrane Library were searched for relevant studies through January 2018. Fourteen articles $(n=7489)$ meeting the inclusion criteria were finally included. Possible effects were calculated using meta-analysis.

Results: The pooled risk ratios (RRs) of late mortality (>6 months) and acute kidney injury after TAVR in frail group were 2.81 (95\% confidence interval $\left.(\mathrm{Cl}) 1.90-4.15, P<.001, \mathrm{I}^{2}=84 \%\right)$ and $1.41\left(95 \% \mathrm{Cl} 1.02-1.94, P=.04, \mathrm{I}^{2}=24 \%\right)$, respectively. Compared with non-frail group, significantly higher incidence of 30-day mortality (RR 2.03, 95\% Cl 1.63-2.54, $\left.P<.001, I^{2}=0 \%\right)$ and life threatening or major bleeding after TAVR (RR 1.48, 95\% Cl 1.20-1.82, $P<.001, \mathrm{I}^{2}=14 \%$ ) was found in frail group. There was no significant association between frailty and incidence of stroke after TAVR (RR 0.93, 95\% Cl 0.53-1.63, $P=.80, I^{2}=0 \%$ ).

Conclusion: Preoperative frailty status is proved to be significantly associated with poor outcomes after TAVR. Our findings may remind doctors in the field of a more comprehensive preoperative evaluation for TAVR candidates. More well-designed and largesample sized prospective studies are further needed to figure out the best frailty assessment tool for patients undergoing TAVR.

Abbreviations: $\mathrm{AKI}=$ acute kidney injury, $\mathrm{BMI}=$ body mass index, $\mathrm{Cl}=$ confidence interval, $\mathrm{CSH} \mathrm{A}=\mathrm{Canadian} \mathrm{Study}$ of $\mathrm{Health}$ and Aging, GA = general anesthesia, LACS = local anesthesia or conscious sedation, PRISMA = Preferred Reporting Items for Systematic Reviews and Meta-Analyses, $\mathrm{RR}=$ risk ratio, TAVR $=$ transcatheter aortic valve replacement, $\mathrm{VARC}=\mathrm{Valve}$ Academic Research Consortium.
\end{abstract}

Keywords: aortic stenosis, complication, frailty, mortality, risk factor, TAVR

\section{Introduction}

The term "frailty' - a syndrome of impaired physiologic reserve and decreased resistance to stressors ${ }^{[1]}$ initially derives from gerontology and is used to estimate health conditions and predict risks of adverse events for old population. In recent years, the use of

\footnotetext{
Editor: Ovidiu Constantin Baltatu.

The authors have no funding and conflicts of interest to disclose.

Supplemental Digital Content is available for this article.

a Department of Anesthesiology, West China Hospital, ${ }^{b}$ Department of Obstetrics and Gynecology, West China Second University Hospital, Sichuan University,

Chengdu, Sichuan, China.

* Correspondence: XiaoYun Yang, Department of Obstetrics and Gynecology, West China Second University Hospital, Sichuan University, Chengdu 610041, China (e-mail: cdyangxiaoyun@163.com); Hai Yu, Department of Anesthesiology, West China Hospital, Sichuan University, Chengdu 610041, China

(e-mail: yuhaishan117@yahoo.com).

Copyright (c) 2018 the Author(s). Published by Wolters Kluwer Health, Inc. This is an open access article distributed under the terms of the Creative Commons Attribution-Non Commercial License 4.0 (CCBY-NC), where it is permissible to download, share, remix, transform, and buildup the work provided it is properly cited. The work cannot be used commercially without permission from the journal.

Medicine (2018) 97:51(e13475)

Received: 8 April 2018 / Accepted: 7 November 2018

http://dx.doi.org/10.1097/MD.0000000000013475
}

frailty assessment has been expanded to the field of preoperative evaluation, especially in high-risk patients undergoing transcatheter aortic valve replacement (TAVR). An objective frailty assessment is recommended by 2 national guidelines to be integrated into pre-TAVR risk evaluation last year. ${ }^{[2,3]}$ Several studies suggest that preoperative frailty status is associated with increased mortality. ${ }^{[4,5]}$ Frail patients undergoing TAVR are more likely to have increased postoperative length of stay and higher incidence of postprocedural complications. ${ }^{[6-8]}$ Acute kidney injury (AKI) is a frequent complication after TAVR, ranging from $8.3 \%$ to $57 \%$ of patients according to the Valve Academic

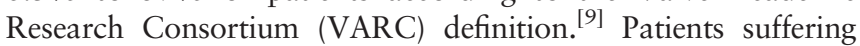
from AKI after TAVR tend to have higher 30-day and late mortality. ${ }^{[10,11]}$ Results of former studies present a higher incidence of AKI after TAVR among frail patients, although most of them do not reach statistical significance. ${ }^{[6,12,13]}$ Moreover, frailty assessment is proved to be a strong predictive indicator for functional outcomes and quality of life in patients undergoing TAVR. ${ }^{[6,14,15]}$

A previous meta-analysis suggests no significant association between frailty status and AKI after TAVR. ${ }^{[16]}$ However, a recent large sample-sized observational study reveals a significantly higher risk of AKI after TAVR in frail patients. ${ }^{[13]}$ It is possible that former studies are underpowered because of a relatively small sample size. In addition, the association between frailty and AKI after TAVR is ill-defined due to the heterogeneity of 
definitions used for AKI and frailty. We noticed that standard endpoint definitions promoted in VARC-2 consensus document were widely used in recent studies to investigate relationship between frailty and outcomes after TAVR. It is apparent that results of these studies possess an improved comparability and interpretability. Here, we conducted this systematic review and meta-analysis to provide an update on relationship between preoperative frailty status and outcomes among patients undergoing TAVR.

\section{Methods}

The research is being reported in line with the PRISMA (Preferred Reporting Items for Systematic Reviews and Meta-Analyses) Guidelines. ${ }^{[17]}$ Ethical approval was not required considering the nature of the study.

\subsection{Study selection}

We searched PubMed, Embase, and the Cochrane Library through January 2018 without language restriction using the key search terms frailty and TAVR. Complementary references were further identified by reviewing relevant papers including review, meta-analysis, and these retrieved references.

The inclusion criteria were as follows: patients undergoing TAVR; participants grouped by preoperative frailty status or with full frailty assessment information; available data with odds ratio, risk ratio (RR), and hazard ratio or sufficient data to calculate them with $95 \%$ confidence interval $(95 \% \mathrm{CI})$; studies reporting clinical outcomes after TAVR; and original articles designed as observational studies (retrospective or prospective cohort studies). Studies without a clear definition of frailty or recording frailty as a continuous variable were also excluded. No limitations were put on age of the participants and routes of operative access.

\subsection{Types of outcome measures and data extraction}

Frailty was recorded as a binary variable. The primary outcomes were late mortality (>6 months) and AKI after TAVR. Postoperative 30-day mortality, stroke and life-threatening or major bleeding were secondary outcomes. Weighted means were calculated based on the product of the number of patients.

Two reviewers (LiLi Huang and XiaoShuang Zhou) screened abstracts identified in the initial search. Once meeting the inclusion criteria, full-text were obtained and then reviewed in duplicate. Disagreements between the reviewers were resolved through consultations with 2 reviewers (XiaoYun Yang and Hai $\mathrm{Yu}$ ). A data abstraction form was used to record the details of study characteristics, including journal, frailty definition, sample size, and so on. The primary outcome was late mortality $(>6$ months) after TAVR. Incidence of AKI and 30-day mortality and after TAVR was secondary outcomes.

\subsection{Quality and bias assessment}

Newcastle-Ottawa quality assessment scale was used to evaluate the quality of cohort studies from the following 3 aspects: selection (4 points); comparability ( 2 points); and outcomes ( 3 points). The score ranges from 0 to 9 points with $\geq 7$ points considered high quality and $<7$ moderate or low quality. ${ }^{[18]}$ Two reviewers performed quality assessments independently and the third reviewer would be consulted to reach consensus. For outcomes with the greatest number of studies, we created a funnel plot and used Egger test to assess publication bias. ${ }^{[19]}$

\subsection{Statistical analysis}

This meta-analysis was performed using Review Manager software 5.3 (RevMan, The Cochrane Collaboration, Oxford, UK). Due to various types of frailty assessment tools and different lengths of follow-up in each study, significant heterogeneity can be expected. For this reason, a random-effect model was applied through the whole calculating process. Point estimates and standard errors derived from included studies were combined by the generic inverse variance method of Der-Simonian and Laird. ${ }^{[20]}$ Heterogeneity among pooled studies was reported as the $\mathrm{I}^{2}$ test, where $\mathrm{I}^{2}>50 \%$ indicated substantial heterogeneity. Sensitivity analysis was further performed to explore possible cause for heterogeneity. A 2-tailed $P$ value of $<.05$ was considered a criterion for statistical significance.

\section{Results}

\subsection{Literature search and study characteristics}

We identified 527 records through our search strategy. Three hundred seventy-two records were screened based on abstracts after duplicates removed. Possibly related articles were then assessed for eligibility via full-text reading. Finally, 14 articles with 7489 participants were included in this meta-analysis. The PRISMA flow diagram depicts selection process (see Fig. 1).

In total, 5 types of frailty definition were used among included studies. Seven studies ${ }^{[6-8,21-24]}$ with 1220 participants defined frailty status by a frailty index score based on several indices, such as weight loss, hand grip strength, gait speed, albumin, and the Katz index of activity of daily living. Four studies ${ }^{[13,25-27]}$ with 4583 participants referred to Canadian Study of Health and Aging (CSHA) Clinical Frailty Scale. One studies combined CSHA Clinical Frailty Scale with several other indices for evaluation. ${ }^{[15]}$ Geriatric Status Scale and Katz index were each applied in 1 study. ${ }^{[12,28]}$ Table 1 shows characteristics of each included study. The proportion of patients identified as frail ranges from $4.6 \%$ to $62.4 \%$. All studies reported either 30 -day mortality or late mortality ( $>6$ months) after TAVR, or both. Six studies $^{[6,7,12,13,21,22]}$ reported details of postprocedural complications based on the consensus guidelines of VARC ${ }^{[29,30]}$ including AKI, stroke, and life threatening or major bleeding.

\subsection{Meta-analysis of effects on primary outcomes}

Compared with non-frail group, the risk of AKI after TAVR was higher in frail group (RR 1.41, 95\% CI 1.02-1.94, $P=.04, \mathrm{I}^{2}=$ $24 \%$ ), as shown in Fig. 2. The relationship between frailty and late mortality after TAVR was investigated in 10 studies $(n=$ 2992). The pooled RR of late mortality in frail group was 2.81 (95\% CI 1.90-4.15, $P<.001, \mathrm{I}^{2}=84 \%$ ) (see Fig. 3). Six of ten studies $(n=1029)$ applied the frailty index score to estimate frailty. The pooled RR of late mortality after TAVR in frail patients based on frailty index score was 2.69 (95\% CI 2.063.50, $P<.001, \mathrm{I}^{2}=0$ ) (see Figure, Supplemental Content 1, http:// links.lww.com/MD/C672).

\subsection{Meta-analysis of effects on secondary outcomes}

Eleven studies $(n=7554)$ quantified the relationship between frailty and 30-day mortality after TAVR. Patients in frail group had a 2.03 -fold increased hazard of 30 -day mortality $195 \% \mathrm{CI}$ 1.63-2.54, $P<.001, \mathrm{I}^{2}=0 \%$ ) (see Figure, Supplemental Content 2, http://links.lww.com/MD/C672). Compared with non-frail 


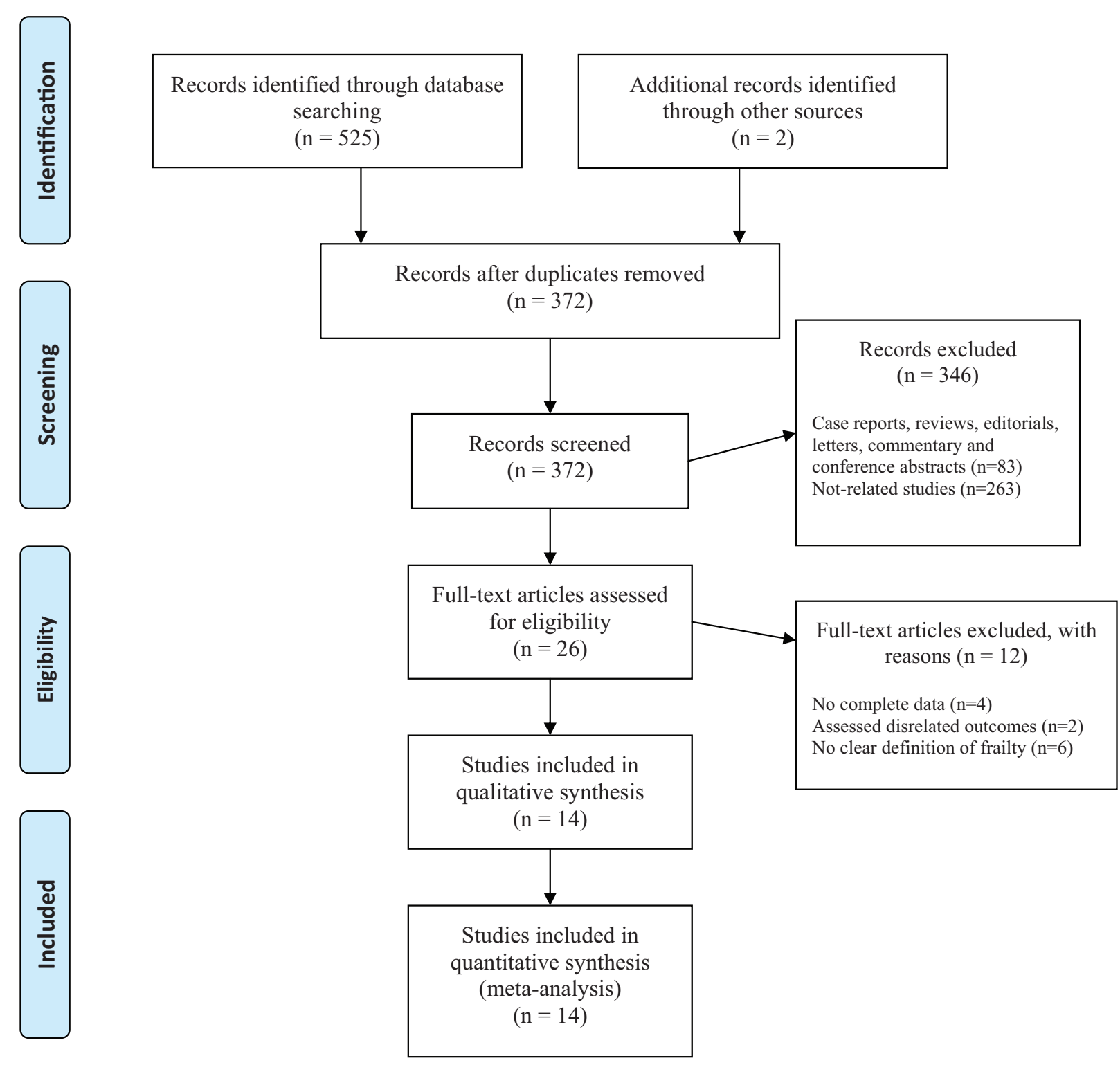

From: Moher D, Liberati A, Tetzlaff J, Altman DG, The PRISMA Group (2009). Preferred Reporting /tems for Systematic Reviews and MetaAnalyses: The PRISMA Statement. PLoS Med 6(7): e1000097. doi:10.1371/journal.pmed1000097

For more information, visit www.prisma-statement.org.

Figure 1. Flow chart. PRISMA flow diagram of study selection process.

group, the risk of life threatening or major bleeding after TAVR was higher in frail group (RR 1.48, 95\% CI 1.20-1.82, $P<.001$, $\mathrm{I}^{2}=14 \%$ ) (see Figure, Supplemental Content 3, http://links.lww. $\mathrm{com} / \mathrm{MD} / \mathrm{C} 672$ ). No significant relationship was found between frailty and incidence of stroke after TAVR (RR 0.93, 95\% CI $0.53-1.63, P=.80, \mathrm{I}^{2}=0 \%$ ) (see Figure, Supplemental Content 4, http://links.lww.com/MD/C672).

\subsection{Study quality and risk of bias}

Thirteen of included studies showed high quality and only one were rated as moderate to low quality. The average score of Newcastle-Ottawa quality assessment is 7 . The study scoring
$<7$ points did not adjust for potential confounders of the relationship between frailty and outcomes. Both funnel plot and Egger tests suggest no significant publication bias on relationship between 30-day mortality and preoperative frailty status among the 11 studies (Egger test for asymmetry $P=.441$ ) (see Figure, Supplemental Contents 5 and 6, http://links.lww.com/MD/ C672).

\section{Discussion}

As far as we know, this meta-analysis is the first available evidence identifying the relationship between frailty and postprocedural complications including stroke and life-threatening or 


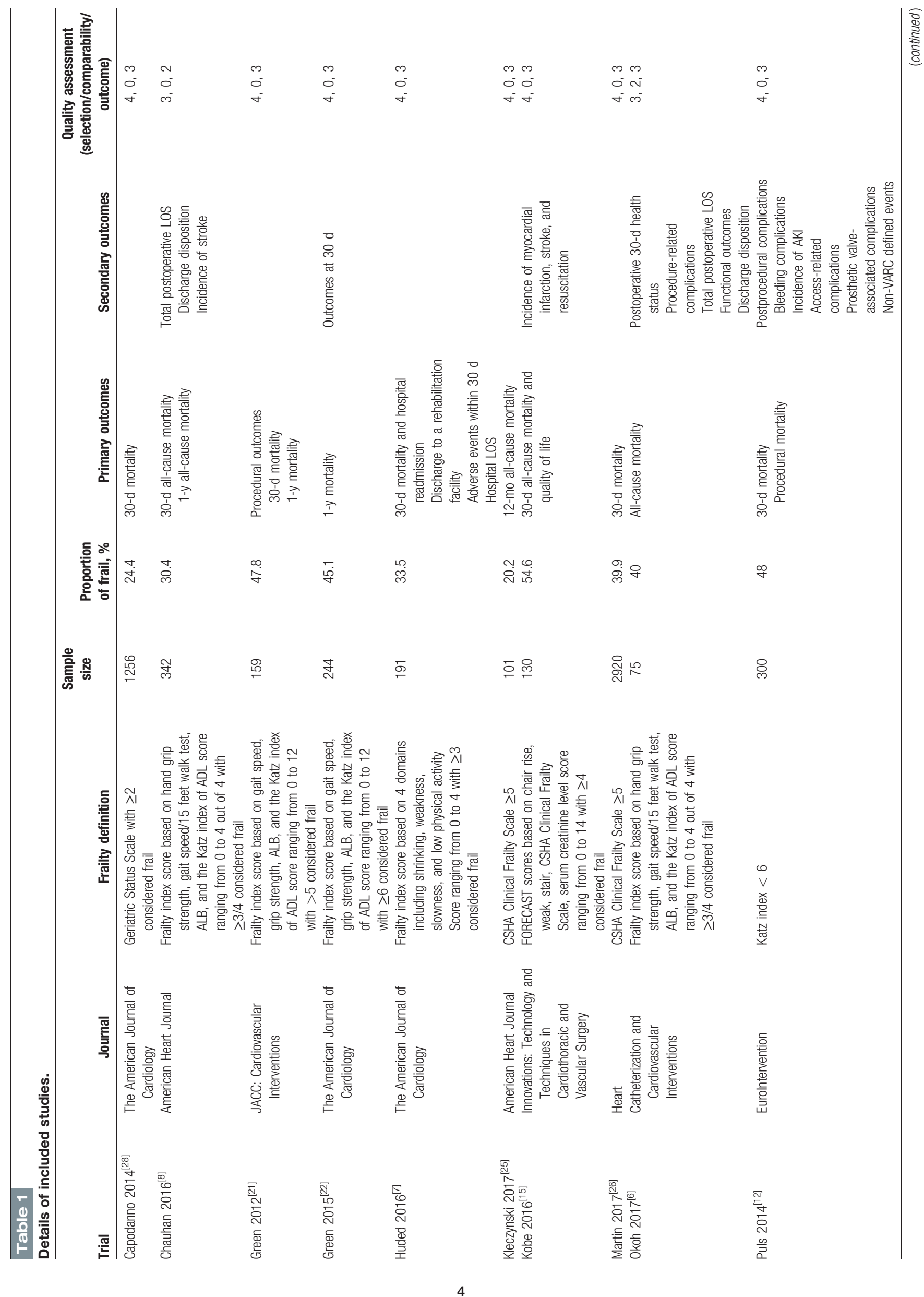




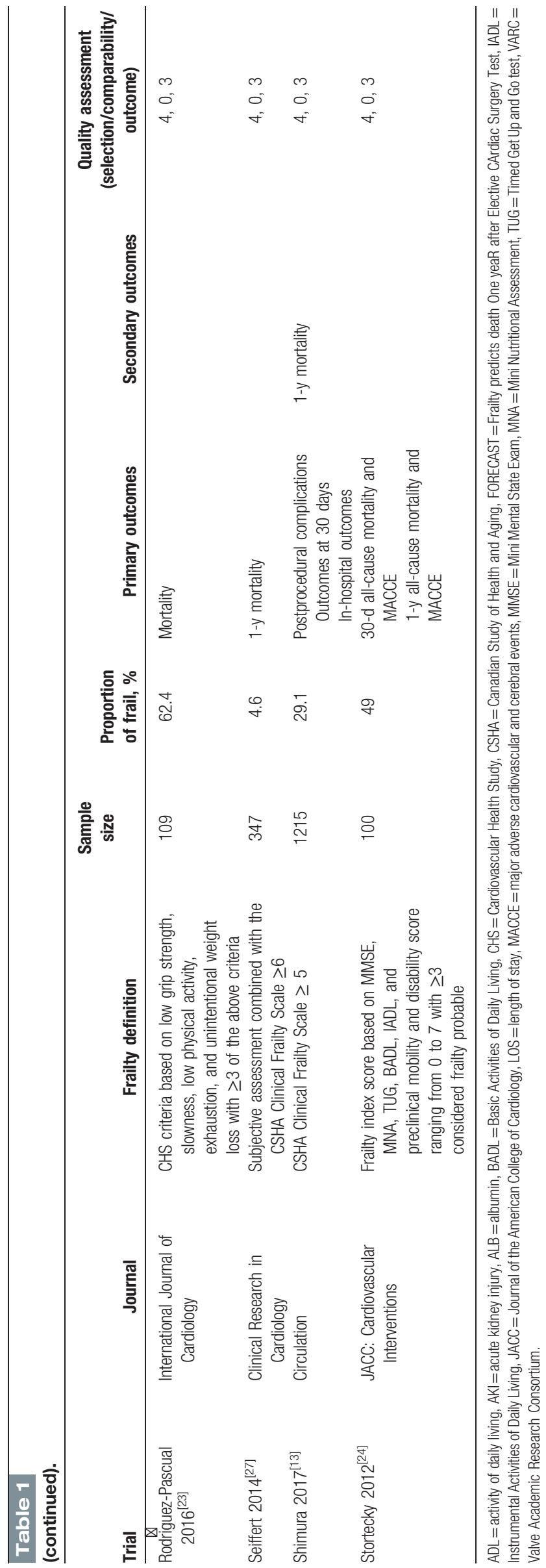

major bleeding. In this systematic review and meta-analysis, we found that the risk of 30-day and late mortality ( $>6$ months) after TAVR was significantly higher in frail group. Preoperative frailty status was demonstrated to be a risk factor of AKI and lifethreatening or major bleeding after TAVR, while incidence of stroke was comparable in 2 groups.

Compared with previous meta-analysis, our research reconfirmed the relationship between preoperative frailty status and increased mortality after TAVR. Frailty assessment appeared to have a good predictive ability on both short- and long-term survivals. The result on relationship of frailty and AKI was not consistent with the study by Thongprayoon et al. ${ }^{[16]}$ Our metaanalysis indicated a 1.41-fold increased risk of AKI after TAVR in frail patients, while no significant association was suggested in their study. It is probably because former studies were underpowered due to a relatively small sample size and various AKI definitions. Besides, we have made several improvements in methodology. First, literature screening process of our study was more precise. For example, body mass index (BMI) is one of additional risk factors indicative of frailty. The study by Yamamoto and colleagues, as stated in the article, only focused on the relation between BMI and clinical outcomes after TAVR and was not related to frailty. ${ }^{[31]}$ Studies like this were filtered out. Second, we excluded studies using subjective frailty assessment, as objective frailty assessment was strongly proposed by national guidelines for TAVR candidates. We aimed to avoid artificial subjective interference and offer reliable hints for future studies.

Although 14 relevant studies were included in our study, only 3 reported on anesthesia. ${ }^{[13,24-25]} \mathrm{A}$ recent study by Husser et a ${ }^{[32]}$ found use of local anesthesia or conscious sedation (LACS) in TAVR is safe, with fewer postprocedural complications and lower early mortality compared with general anesthesia (GA). We can assume that anesthesia may play an important role in TAVR patients with preoperative frailty status. But it is difficult to do further analysis based on different anesthesia due to limited data.

Importance of frailty in pre-TAVR assessment is being emphasized and gradually used in addition to the current risk scores such as the EuroSCORE (European System for Cardiac Operative Risk Evaluation) and the Society of Thoracic Surgeons to improve predictive accuracy. ${ }^{[3-36]}$ However, promotion of its use in clinical practice is not easy due to the lack of a unanimous frailty assessment tool. There is no consensus on the most appropriate assessment tool for TAVR candidates. It is noteworthy that a recent study comparing the predictive value of 7 different frailty scales for older adults undergoing conventional surgery or TAVR found that a brief 4-item scale encompassing lower-extremity weakness, cognitive impairment, anemia, and hypoalbuminemia outperformed other frailty scales. ${ }^{[37]}$ Testing effectiveness of different frailty measurements to work out an optimum assessment tool for TAVR candidates may be a research hotspot in the future.

There were also some limitations. First, this was a metaanalysis of observational studies and inherent limitations do exist due to the nature of these studies. Second, some of included studies did not make adjustments for potential confounders in the relationship between frailty and outcomes such as age and gender. Third, the measurements for frailty were diverse among included studies. We further did sensitivity analysis to minimize the bias.

In summary, existing evidences show a specific correlation between preoperative frailty status and outcomes after TAVR. It 


\begin{tabular}{|c|c|c|c|c|c|c|c|c|c|}
\hline Study or Subgroup & \multicolumn{2}{|c|}{ Frail Group } & \multicolumn{2}{|c|}{ Non-frail Group } & Weight & $\begin{array}{c}\text { Risk Ratio } \\
\text { M-H, Random, } 95 \% \mathrm{Cl} \\
\end{array}$ & \multicolumn{3}{|c|}{$\begin{array}{c}\text { Risk Ratio } \\
\text { M-H, Random, } 95 \% \mathrm{Cl}\end{array}$} \\
\hline Green 2012 & 3 & 76 & 3 & 83 & $4.0 \%$ & $1.09[0.23,5.25]$ & & & \\
\hline Okoh 2017 & 2 & 30 & 0 & 45 & $1.1 \%$ & $7.42[0.37,149.32]$ & & & \\
\hline Puls 2014 & 50 & 144 & 47 & 156 & $49.3 \%$ & $1.15[0.83,1.60]$ & & . & $=$ \\
\hline Shimura 2017 & 47 & 353 & 67 & 862 & $45.6 \%$ & $1.71[1.21,2.44]$ & & & $\rightarrow-$ \\
\hline Total $(95 \% \mathrm{Cl})$ & & 603 & & 1146 & $100.0 \%$ & $1.41[1.02,1.94]$ & & & \\
\hline Total events & 102 & & 117 & & & & & & \\
\hline \multicolumn{7}{|c|}{$\begin{array}{l}\text { Heterogeneity: } \mathrm{Tau}^{2}=0.03 ; \mathrm{Chi}^{2}=3.95, \mathrm{df}=3(\mathrm{P}=0.27) ; \mathrm{I}^{2}=24 \% \\
\text { Test for overall effect: } \mathrm{Z}=2.09(\mathrm{P}=0.04)\end{array}$} & 0.2 & $\begin{array}{c}0.5 \\
\text { Frail group }\end{array}$ & ${ }_{\text {Non-frail group }}^{2}$ \\
\hline
\end{tabular}

Figure 2. Forest plot of included studies comparing the risk of AKI after TAVR in frail versus non-frail patients. Patients in frail group were significantly at higher risk of AKI after TAVR, compared with non-frail patients. Squares represent RR and horizontal lines represent the 95\% Cl for each study. A diamond data marker represents the pooled $\mathrm{RR}$ across studies. AKI=acute kidney injury, $\mathrm{Cl}=$ confidence interval, $\mathrm{RR}=$ risk ratio, TAVR=transcatheter aortic valve replacement.

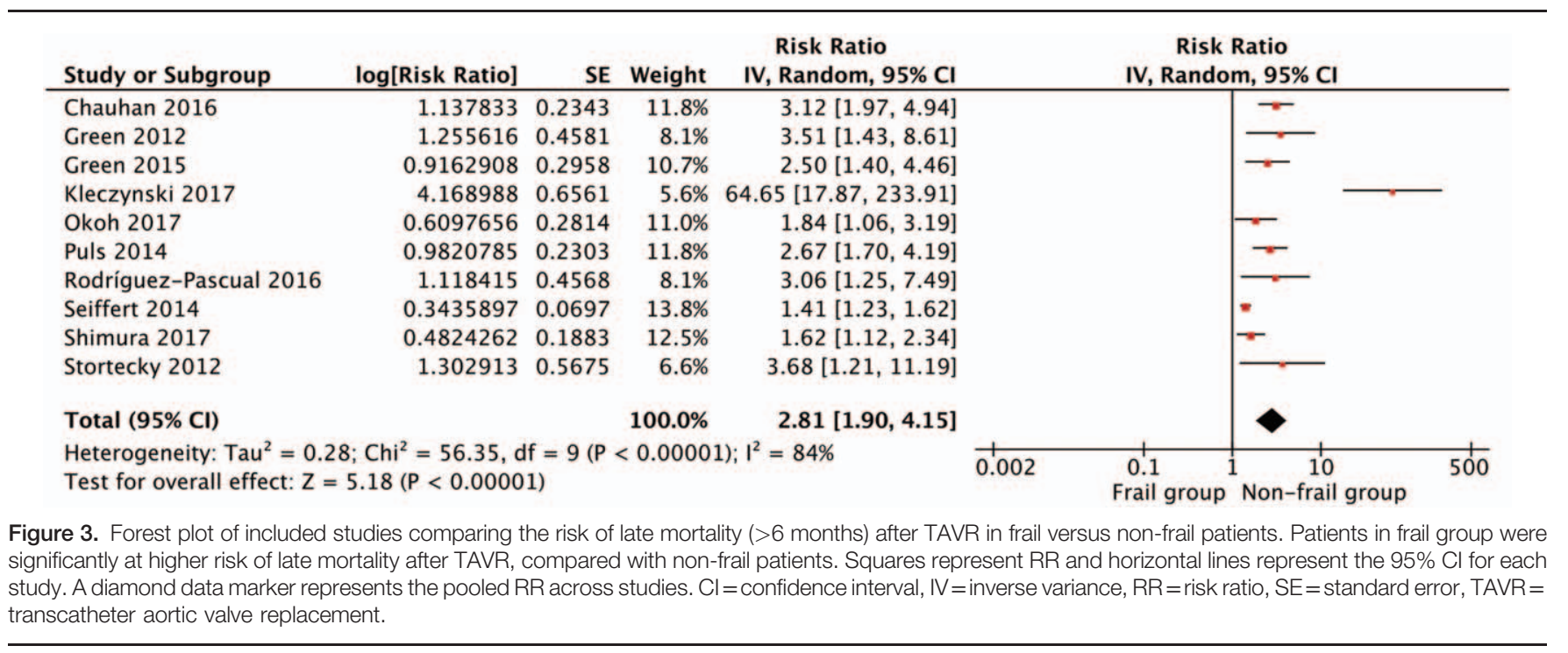

is imperative to find effective clinical interventions for this setting. Prehabilitation to reduce frailty has been reported to improve postsurgical outcomes in a variety of populations. ${ }^{[38,39]}$ In addition, we think an individual anesthesia regimen may be benefit to frail patients. Future studies may compare LACS and GA in frail patients and provide evidence for clinicians.

\section{Conclusion}

Preoperative frailty status is proved to have a strong predictive ability of both 30-day and late mortality after TAVR. The findings from our study also suggest significant associations between frailty and postprocedural complications including AKI and life threatening or major bleeding. Well-designed and largesample sized prospective studies are further needed to find out the best frailty assessment tool for TAVR candidates and researchers should as well pay attention to exploring effective interventions for frail patients.

\section{Author contributions}

Conceptualization: LiLi Huang, XiaoYun Yang, Hai Yu. Data curation: LiLi Huang, XiaoShuang Zhou, Hai Yu.
Formal analysis: LiLi Huang, XiaoShuang Zhou, XiaoYun Yang, Hai Yu.

Methodology: LiLi Huang, XiaoShuang Zhou, XiaoYun Yang, Hai Yu.

Supervision: XiaoYun Yang.

Validation: LiLi Huang, Hai Yu.

Writing - original draft: LiLi Huang, Hai Yu.

Writing - review \& editing: LiLi Huang, XiaoYun Yang, Hai Yu.

\section{References}

[1] Fried LP, Hadley EC, Walston JD, et al. From bedside to bench: research agenda for frailty. Sci Aging Knowledge Environ 2005;2005:e24.

[2] Otto CM, Kumbhani DJ, Alexander KP, et al. 2017 ACC expert consensus decision pathway for transcatheter aortic valve replacement in the management of adults with aortic stenosis: a report of the American College of Cardiology Task Force on Clinical Expert Consensus Documents. J Am Coll Cardiol 2017;69:1313-46.

[3] Nishimura RA, Otto CM, Bonow RO, et al. 2017AHA/ACC focused update of the 2014 AHA/ACC Guideline for the Management of Patients With Valvular Heart Disease: a report of the American College of Cardiology/American Heart Association Task Force on Clinical Practice Guidelines. J Am Coll Cardiol 2017;70:252-89.

[4] Kamga M, Boland B, Cornette P, et al. Impact of frailty scores on outcome of octogenarian patients undergoing transcatheter aortic valve implantation. Acta Cardiol 2013;68:599-606. 
[5] Assmann P, Kievit P, van der Wulp K, et al. Frailty is associated with delirium and mortality after transcatheter aortic valve implantation. Open Heart 2016;3:e00478.

[6] Okoh AK, Chauhan D, Kang N, et al. The impact of frailty status on clinical and functional outcomes after transcatheter aortic valve replacement in nonagenarians with severe aortic stenosis. Catheter Cardiovasc Interv 2017;90:1000-6.

[7] Huded CP, Huded JM, Friedman JL, et al. Frailty status and outcomes after transcatheter aortic valve implantation. Am J Cardiol 2016;117: 1966-71.

[8] Chauhan D, Haik N, Merlo A, et al. Quantitative increase in frailty is associated with diminished survival after transcatheter aortic valve replacement. Am Heart J 2016;182:146-54.

[9] Elhmidi Y, Bleiziffer S, Deutsch MA, et al. Acute kidney injury after transcatheter aortic valve implantation: incidence, predictors and impact on mortality. Arch Cardiovasc Dis 2014;107:133-9.

[10] Saia F, Ciuca C, Taglieri N, et al. Acute kidney injury following transcatheter aortic valve implantation: incidence, predictors and clinical outcome. Int J Cardiol 2013;168:1034-40.

[11] Nunes Filho ACB, Katz M, Campos CM, et al. Impact of acute kidney injury on short- and long-term outcomes after transcatheter aortic valve implantation. Revista espanola de cardiologia (English ed) 2018;doi: 10.1016/j.rec.2017.11.024. Accessed January 24, 2018.

[12] Puls M, Sobisiak B, Bleckmann A, et al. Impact of frailty on short- and long-term morbidity and mortality after transcatheter aortic valve implantation: risk assessment by Katz Index of activities of daily living. EuroIntervention 2014;10:609-13.

[13] Shimura T, Yamamoto M, Kano S, et al. Impact of the clinical frailty scale on outcomes after transcatheter aortic valve replacement. Circulation 2017;135:2013-24.

[14] Schoenenberger AW, Stortecky S, Moser A, et al. Predictors of functional decline in elderly patients undergoing transcatheter aortic valve implantation (TAVI). Eur Heart J 2012;33:587.

[15] Kobe AR, Meyer A, Elmubarak H, et al. Frailty assessed by the FORECAST is a valid tool to predict short-term outcome after transcatheter aortic valve replacement. Innovations (Phila) 2016;11: $407-13$.

[16] Thongprayoon C, Cheungpasitporn W, Thamcharoen N, et al. Association of frailty status with acute kidney injury and mortality after transcatheter aortic valve replacement: a systematic review and metaanalysis. PLOS ONE 2017;12:e0177157.

[17] Moher D, Liberati A, Tetzlaff J, et al. Preferred reporting items for systematic reviews and meta-analyses: the PRISMA statement. PLoS Med 2009;6:e1000097.

[18] Wells G, Shea B, O'Connell D, et al. The Newcastle-Ottawa Scale (NOS) for assessing the quality of nonrandomised studies in meta- analyses Ottawa [cited 2018 April 8 2018]. Available from: http://www.ohri.ca/ programs/clinical_epidemiology/oxford.asp.

[19] Egger M, Davey Smith G, Schneider M, et al. Bias in meta-analysis detected by a simple, graphical test. BMJ (Clin Res ed) 1997;315:629-34.

[20] DerSimonian R, Laird N. Meta-analysis in clinical trials revisited. Contemp Clin Trials 2015;45:139-45.

[21] Green P, Woglom AE, Genereux P, et al. The impact of frailty status on survival after transcatheter aortic valve replacement in older adults with severe aortic stenosis: a single-center experience. JACC Cardiovasc Interv 2012;5:974-81.

[22] Green P, Arnold SV, Cohen DJ, et al. Relation of frailty to outcomes after transcatheter aortic valve replacement (from the PARTNER trial). Am J Cardiol 2015;116:264-9.
[23] Rodriguez-Pascual C, Paredes-Galan E, Ferrero-Martinez AI, et al. The frailty syndrome and mortality among very old patients with symptomatic severe aortic stenosis under different treatments. Int J Cardiol 2016;224:125-31.

[24] Stortecky S, Schoenenberger AW, Moser A, et al. Evaluation of multidimensional geriatric assessment as a predictor of mortality and cardiovascular events after transcatheter aortic valve implantation. JACC Cardiovasc Interv 2012;5:489-96.

[25] Kleczynski P, Dziewierz A, Bagienski M, et al. Impact of frailty on mortality after transcatheter aortic valve implantation. Am Heart J 2017;185:52-8.

[26] Martin GP, Sperrin M, Ludman PF, et al. Novel United Kingdom prognostic model for 30-day mortality following transcatheter aortic valve implantation. Heart 2018;104:1109-16.

[27] Seiffert M, Sinning JM, Meyer A, et al. Development of a risk score for outcome after transcatheter aortic valve implantation. Clin Res Cardiol 2014;103:631-40.

[28] Capodanno D, Barbanti M, Tamburino C, et al. A simple risk tool (the OBSERVANT score) for prediction of 30-day mortality after transcatheter aortic valve replacement. Am J Cardiol 2014;113:1851-8.

[29] Kappetein AP, Head SJ, Genereux P, et al. Updated standardized endpoint definitions for transcatheter aortic valve implantation: the Valve Academic Research Consortium-2 consensus document (VARC2). Eur J Cardiothorac Surg 2012;42:S45-60.

[30] Leon MB, Piazza N, Nikolsky E, et al. Standardized endpoint definitions for transcatheter aortic valve implantation clinical trials: a consensus report from the Valve Academic Research Consortium. J Am Coll Cardiol 2011;57:253-69.

[31] Yamamoto M, Hayashida K, Watanabe Y, et al. Effect of body mass index $<20 \mathrm{~kg} / \mathrm{m}^{2}$ on events in patients who underwent transcatheter aortic valve replacement. Am J Cardiol 2015;115:227-33.

[32] Husser O, Fujita B, Hengstenberg C, et al. Conscious sedation versus general anesthesia in transcatheter aortic valve replacement: the German Aortic Valve Registry. JACC Cardiovasc Interv 2018;11: 567-78.

[33] Dewey TM, Brown D, Ryan WH, et al. Reliability of risk algorithms in predicting early and late operative outcomes in high-risk patients undergoing aortic valve replacement. J Thorac Cardiovasc Surg 2008; 135:180-7.

[34] Osswald BR, Gegouskov V, Badowski-Zyla D, et al. Overestimation of aortic valve replacement risk by EuroSCORE: implications for percutaneous valve replacement. Eur Heart J 2009;30:74-80.

[35] Grossi EA, Schwartz CF, Yu PJ, et al. High-risk aortic valve replacement: are the outcomes as bad as predicted? Ann Thorac Surg 2008;85: 102-6.

[36] Schoenenberger AW, Moser A, Bertschi D, et al. Improvement of risk prediction after transcatheter aortic valve replacement by combining frailty with conventional risk scores. JACC Cardiovasc Interv 2018; 11:395-403.

[37] Afilalo J, Lauck S, Kim DH, et al. Frailty in older adults undergoing aortic valve replacement: the FRAILTY-AVR study. J Am Coll Cardiol 2017;70:689-700.

[38] Rumer KK, Saraswathula A, Melcher ML. Prehabilitation in our most frail surgical patients: are wearable fitness devices the next frontier? Curr Opin Organ Transplant 2016;21:188-93.

[39] Waite I, Deshpande R, Baghai M, et al. Home-based preoperative rehabilitation (prehab) to improve physical function and reduce hospital length of stay for frail patients undergoing coronary artery bypass graft and valve surgery. J Cardiothorac Surg 2017;12:91. 